\title{
Comments on Einstein's Explanation of Electrons, Photons, and the Photo-Electric Effect
}

\author{
Salama Abdelhady \\ Professor of Energy Systems \\ Department of Mechanical Engineering, CIC, Cairo, Egypt \\ E-mail: salama_hady@cic-cairo.com
}

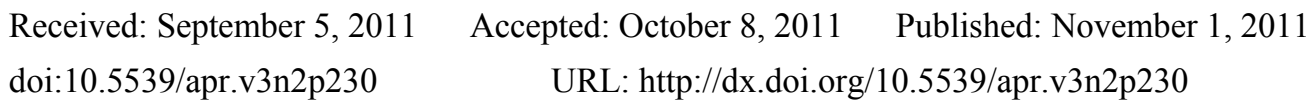

\begin{abstract}
:
According to an entropy approach and by reviewing the similarity between laws characterizing the flow of heat and electric charges, electric charges were defined as electromagnetic waves that possess an electrical potential or simply as ionized photons. Accordingly, the flow of electrons was defined as a simultaneous flow of particulate energy and wave energy. Such definitions led to clear the confusions of duality properties of electrons and light waves, conflicts in the SI system of units and to explain the difference between the calculated drift speed of electrons and the speed of electricity or charges in conductors. However, Einstein considered the electron to be a negative charge of unknown nature during his analysis of the photoelectric effect. Einstein presented his hypothesis that light may behave as a particle to find a plausible explanation of the photoelectric effect. He found the measured cutoff frequency of light below which light might not eject electrons from metal-surfaces in photocells regardless of how much light is shone on the surface as a proof of truth of his hypothesis. Such frequency may be explained also, according to the previously introduced definitions, as the minimum energy quanta that may gain a quantized potential in photocells. This explanation may find plausible explanations of the found measurements of the photoelectric effects for different metal surfaces, stopping voltages into photocells and the photoelectric effect of X-rays. Einstein's hypothesis of the photoelectric effect failed to find plausible explanations of such measurement results. According to the principles of conservation of momentum, there is a minimum quantity of photons that may lead to bouncing an atomic electron out of its orbit. So, considering Einstein's theory as a perfect hypothesis that explains the photoelectric effect may be a misconception.
\end{abstract}

Keywords: Electrons, Photons, Photoelectric effect, Light, Charge, Electron-volt, Tthreshold frequency

\section{Introduction}

According to literature of physics; the flow of electric charges is considered as flow of electrons (D. Haaiday,et al., 2004). Such definition was based on an analysis of results of the famous J.J. Thompson's cathode-ray experiment (D. L. Anderson, 2004) that was conducted more than a century ago. It can be proved that defining the electron as a particle and as a charge would lead directly to the duality property of electrons (S. Abdelhady, 2011). Unfortunately, Einstein's analysis of the photoelectric-effect depends directly on such definition of electrons (D.J. Griffiths, 2004).

According to an entropy approach and a held experiment in addition to reviewing the similarity between heat and electric charges, it was possible to postulate in previous papers a new definition of the flow of electric charges as a flow of electromagnetic waves that have a specific electric potential or simply the charge can be postulated as ionized photons (S. Abdelhady, 2010, pp.162-166). Such approach cleared, as proved in previous articles, confusions concerning the nature of the electric charge (S. Abdelhady, 2010, pp. 677-678), the duality property of electrons and light waves (S. Abdelhady, 2011) and conflicts in the SI system of units (S. Abdelhady, 2010, pp. 549-556). According to the postulated definitions, the photoelectric effect was explained as ionizing the incident light waves due to its reflection on an ionized metal surface or that the light waves are converted into electric charges by gaining an electric potential in the photocell's electric field (S. Abdelhady, 2011).

However, Einstein explained the photoelectric effect as it occurs when light hits a metallic surface in a photocell and ejects electrons (W. Sinnema, 1979). Such explanation was the origin of Einstein's theory of light's particulate 
and the quantum mechanics (Albert Einstein, 1905). In fact, explaining theory in this way gave Einstein the Nobel Prize - not, surprisingly, for his theory of relativity. He showed that light is made of photons, which are particles (W.M. Yao, 2006). Einstein supposed that the photoelectric effect occurs when the photon transfers enough energy to eject an electron from an atom in a metallic surface regardless of how much light is shone on the surface. His approach to prove this theory depended on the experimentally found cutoff frequency below which the electrons was not ejected (The Nobel Prize in Physics 1921).

In this article, it is analyzed the Einstein's definitions of electrons and photons that led him to such explanation of the photoelectric effect. Then the analysis compares the success of Einstein's definitions to the newly postulated definitions in finding plausible explanations of measurement results in photocells. Truth of the definition of the flow of electric charge as a flow of electromagnetic waves is firstly proved in finding a plausible explanation of the difference between the calculated velocities of flow of electrons in conductors according to literature of physics, in the order of $\mathrm{mm} / \mathrm{hr}$, and the measured velocity of flow of electric charges in such conductors in the order of $\mathrm{km} / \mathrm{s}$ (http://www.physchem.co.za/OB12-ele/photoelectric.htm). Similarly, the definitions of the cutoff frequency, as defined by Einstein, will be compared to that concluded from the followed entropy approach. According to the followed entropy approach, the cutoff frequency corresponds to the minimum quanta of light energy that may gain a quantized potential due its reflection on the ionized metal surface in the photocell. The truth of this postulate will be proved by reviewing the results of an experimental arrangement similar to that of the cathode ray tube but the cathode was not heated nor had a large bias potential (G. Keiser, 2000). In this experiment, when light impinged upon the surface of the cathode, a flow of electric charges was ejected from the cathode and picked up by the anode (G. Keiser, 2000). If an opposing bias voltage was established, a certain threshold potential was found above which no current would be measured. This was called the stopping voltage. It will be discussed in this paper how the presented analysis of photoelectric effect as ionizing of the incident light waves finds plausible explanation of such stopping voltage. The results of measuring the photoelectric effect by X-rays are discussed in the light of Einstein hypothesis and the followed approach. It will be seen how the incident X-rays are reflected at specific quantized potentials that depend on the frequency of the incident waves (http://www.docstoc.com/docs/80625378/X-Ray-Photoelectron-Spectroscopy-XPS). Such results represent a proof that the light photons are converted into energy quanta or electric charges of specified potentials that depend on the frequency of the incident light. However, it will be shown also how Einstein's explanation of the photoelectric effect as ejection of electrons fails to find plausible explanations of such experimental results.

Compton scattering that defines the interaction between the incident photons and the atomic electrons as collision of the photon elastically with an atomic electron will be analyzed (A. H. Compton, 1923). The analysis will show the importance of transferring both momentum and energy to the ejected electrons to prove the energy is not the sole factor in ejecting electrons but the momentum should be considered too. Applying the principles of momentum conservation, it will be found that the momentum of an atomic electron is equivalent to the momentum of more than one million photons if the photon is considered as a mass particle. Accordingly, it will be discussed the criticism in Einstein's hypothesis that considers photons of frequency higher than the cutoff frequency are able to eject atomic electrons irrespective of their numbers.

\section{Nature of the Electric Charges}

Heat is defined as a form of energy that possesses a potential, temperature, and is flowing as electromagnetic waves. According to the similarity of the laws characterizing the flow of heat and electric charges, results of held experiments and by following an entropy approach, it was possible to prove that the electric charge is also a form of energy that possesses an electric potential and is flowing as electromagnetic waves (S. Abdelhady, 2010, pp. 162-166). According to the analogy between the laws characterizing the flow of heat and flow of electric charge, it was possible to derive the following fundamental equation of thermodynamics that embraces electric charges and magnetic flux and expresses the increase of internal energy of a closed system " $\mathrm{dU}$ " in terms of these energies as follows (S. Abdelhady, 2010, pp. 162-166):

$$
\mathrm{dU}=\mathrm{T}(\mathrm{dS})_{\mathrm{th}}+\mathrm{E}(\mathrm{dS})_{\mathrm{el}}+\mathrm{H}(\mathrm{dS})_{\mathrm{mag}}-\mathrm{pdV}+\sigma \mathrm{d} \Omega+\sum \mu_{\mathrm{i}} \mathrm{dn} n_{\mathrm{i}}
$$

According to equation (1), the term $\mathrm{T}(\mathrm{dS})_{\mathrm{th}}$ expresses the heat flowing to a system as the product of the temperature "T"and increase in the system's entropy " $(\mathrm{dS})_{\mathrm{th}}$ ", the term "E $(\mathrm{dS})_{\mathrm{el}}$ " expresses the electric charge flowing as the product of the electrical potential "E" and increase in system's entropy "(dS)el" and the term "H $(\mathrm{dS})_{\mathrm{mag}}$ " expresses the magnetic flux flowing as the product of the magnetic potential " $H$ " and increase in system's entropy "(dS) mag". Heat can be represented in a T-s diagram as the integral of the elemental area 
$T(\mathrm{dS})_{\text {th }}$. By analogy to heat, it is possible to represent also the flowing charge in a similar E-s diagram as the integral (S. Abdelhady, 2010, pp. 162-166):

$$
Q_{\text {elect }}=\int E(\mathrm{dS})_{\text {elect }}
$$

The electromagnetic waves are characterized by Maxwell's equations. However, the time in Maxwell's equation was replaced by entropy which is a property of time only as entropy is generally understood to signify an inherent tendency towards disorganization that depends on time alone (http://www.marxist.com/science-old/arrowoftime.html). Accordingly entropy represents, in general, the arrow of time as it was introduced in equation (3) and (4) (H. D. Zeh, 1990). Such transformation leads to the following solution of Maxwell's equation that may express the flow of electric charges into space-entropy coordinates as follows (S. Abdelhady, 2010, pp. 162-166):

$$
\begin{gathered}
E(r, s)=E \cos (k r+\omega s+\varphi)+/-\Delta \overline{\mathrm{E}} \\
H(r, s)=H \cos (k r+\omega s+\varphi)
\end{gathered}
$$

Where $\mathrm{r}$ indicates the space coordinate, $\mathrm{s}$ indicates the entropy coordinate, $\omega$ represents the angular speed of the wave and $\varphi$ is a phase shift (S. Abdelhady, 2010, pp. 162-166).

In this solution, the electric component of the wave is oscillating around a non-zero value $(\Delta \overline{\mathrm{E}})$ that determines the potential of the electric charge. The electric charge is determined by area swept by the wave in the E-s diagram according to equation (2). A graphical representation of the solution expressed by equations (3) and (4) is seen in Figure 1. It is the case of flow of a negative charge or a charge of negative potential, $-\Delta \overline{\mathrm{E}}$. Such approach can be considered as a postulate that may offer plausible explanation for the colored charges which may have different values of the electric potentials $\Delta \overline{\mathrm{E}}$ and charge as determined by the swept areas (S. Abdelhady, 2010, pp. 162166).

Heat flow is defined as the product of the temperature times the entropy flow (.C. Yunus and A. B. Michael, 2006). By analogy, the rate of flow of electric charge can be expressed as the product of electric potential "E" times the rate of entropy flow "S'" (S. Abdelhady, 2010, pp. 162- 166):

$$
\dot{\mathrm{Q}}_{\mathrm{elect}}=\mathrm{E} * \dot{\mathrm{S}}
$$

According to equation (6), the unit of $\dot{S}$ is in Watt/Volt.

\section{The Ammeter's Readings}

Reviewing the Coulomb and Ampere as units of the electric charge in the SI system of units, it can be found that such units are sources of confusion as it is not necessary to introduce such units at all (S. Abdelhady, 2010, pp. 677-678). According to the main laws of electricity, electric power is defined as a product of the ammeter's reading " $\dot{A}$ " times the electric potential "E" (D. Haaiday, et al., 2004). Hence, units of the ammeter's readings " $A$ " should be the unit of power per unit potential or in $\frac{\text { Watt }}{\text { volt }}$. However, the unit of such reading is traditionally expressed as Ampere or $\frac{\text { Coulomb }}{\text { second }}$. Hence, the ammeter's reading is expressed, according to the SI system of units, by two equivalent units: ${ }^{W a t t} /$ Volt and Coulomb/second. This conclusion can be expressed mathematically by the following equation (S. Abdelhady, 2010, pp. 549-556):

$$
\dot{A}=\frac{\dot{W}}{E}=\frac{\text { Joul } / \text { sec }}{\text { volt }}=\frac{\text { Coulomb }}{\text { second }},
$$

$\dot{A}$ is the ammeter readings, $\dot{W}$ is the electric power in Watt and $E$ represents the electric potential in volts. According to equation (7); it is possible to write the following identity: 


$$
\text { Coulomb } \equiv \text { joule } / \text { volt }
$$

However, this unit "joule/volt" is a unit of entropy as can be concluded from equation (6). So, the coulomb cannot be considered as a unit of electric charge or electric energy but it is a unit of entropy that flows during electricity flow. In other words, the unit of the electrical charge should be joule which is the defined unit of electric energy. So, the ammeter does not measure the rate of flow of electricity but it measures actually the flow rate of the electric entropy $\dot{S}_{\text {elect }}$ in $\mathrm{Watt} / \mathrm{Vol}$.

In a recent paper (S. Abdelhady, 2010, pp. 549-556), it was found that introducing the Coulomb as a fundamental unit in the SI system of units was the source of confusion. By eliminating such a unit, it was possible to introduce a new system of units that is free from many conflicts of the SI system (S. Abdelhady, 2010, pp. 549-556).

\section{The Electron}

According to the literature, the electron is considered as a particle of mass $9.109382 \times 10^{-31} \mathrm{~kg}$ and as an electric charge which is stated as $1.602 \times 10^{-19}$ Coulomb (D. Haaiday,et al., 2004). Such a definition of the electron as a particle and as a charge led directly to the confusing duality property of electrons that the electron may behave as a particle or a wave where the charge is actually a wave (S. Abdelhady, 2010, pp. 162-166). According to a previous analysis that relied on an entropy approach, the flow of electrons is postulated as a simultaneous flow of particles and electromagnetic waves (S. Abdelhady, 2011). This electron's energy is in the form of electromagnetic waves that can be calculated as the sum of its associated electric, kinetic and magnetic energies (S. Abdelhady, 2011).

$$
E_{w}=K \cdot E \cdot+E \cdot E .+M \cdot E
$$

For an electron flowing at half speed of light, its kinetic energy can be found as follows:

$$
\text { K.E. }=1 / 2 m_{o} v^{2}=1 / 29.10953 * 10^{-31} *\left(\frac{2.99 * 10^{8}}{2}\right)^{2}=1.016 * 10^{-14} \mathrm{Joule}
$$

However, the electron as a charged particle of magnetic spinning has also an electric energy of $4.1032 * 10^{-14}$ Joule and magnetic energy of $4.0842 * 10^{-14}$ Joule (S. Abdelhady, 2010, pp. 162-166). Substituting such data into equation (9), the total wave energy associated by an electron that flows at half the velocity of light, is found as follows:

$$
E_{w}=(1.016+4.1032+4.0842) * 10^{-14}=9.2034 * 10^{-14} \mathrm{Joule}
$$

A relativistic relation between the rest mass of a particle " $m_{0}$ " and its relativistic mass " $m_{e}$ " is found as follows (.C. Yunus and A. B. Michael, 2006):

$$
E_{w}=m_{e} c^{2}-m_{0} c^{2}
$$

Accordingly, the flow of electrons may be defined as a simultaneous flow of particulized energy or rest electron's energy " $m_{0} c$ " " and wave energy " $E_{w}$ ". Hence, the total energy of a flowing electron " $E_{e}$ " can be calculated for an electron moving at half speed of light as follows (S. Abdelhady, 2011):

$$
E_{e}=E_{o}+E_{w}=(8.19+9.2034) * 10^{-14}=17.3934 * 10^{-14} \mathrm{Joule}
$$

Therefore the ratios of the two components of such electron's energy; rest energy and its associated wave energy, to the whole electron's energy " $\mathrm{E}_{\mathrm{e}}$ ", can be calculated as follows:

$$
\mu_{o}=\frac{E_{o}}{E_{e}}=0.472
$$




$$
\mu_{w}=\frac{E_{w}}{E_{e}}=0.528
$$

The values $\mu_{\mathrm{o}}$ and $\mu_{\mathrm{w}}$ can be considered as memberships of the particle and the wave components of energy in an electron's flow. In other words, the behavior of electron's flow as a particle and as a wave depends on the relative values of these two ratios which are functions of the electron's speed and charge (D.J. Griffiths, 2004). This conclusion can be considered as an explanation of the de Broglie proposition that all particles may have electromagnetic waves of specific wavelengths (D. Haaiday, et al., 2004). Actually, the energy associated by each flowing particle has a specific wavelength that depends on the forms of the associated kinetic energy which is function of its velocity (E.J.S. Fonseca, et al.).

According to previous literature (D. Haaiday, et al., 2004), the flow of charge is supposed to be tied by the flow of the charge carriers. So, if the charge carriers, as electrons, have a charge (q) and move through the material at a velocity (v), this velocity is considered to be electron's drift velocity that determines also the velocity of the charge. Within the material not all the charged particles are free to move. So, the carrier density (n) is the number of charge carriers free to move per cubic meter. The electric current was defined as the total amount of electrons passing a given cross-section (A) per second which can be estimated by the following transport equation (W. Sinnema, 1979):

$$
I=n A v q
$$

For a current of 10 Ampere and for $\mathrm{n}=10^{29}$ electrons per $\mathrm{m}^{3}$ in metals, $\mathrm{q}=1.6 \times 10^{-19}$ and in a typical 12 gauge copper wire of cross-sectional area $A=3.3 \times 10^{-6} \mathrm{~m}^{2}$, the drift speed or velocity of charge carriers can be estimated from equation (13) as follows: $\mathrm{v}=2 \times 10^{-4} \mathrm{~m} / \mathrm{s}$.

However, it is impossible to believe such a velocity determines the velocity of the electric current in copper wires that are traditionally used in wiring of buildings. Professor Charles Wheatstone (Bray John, 1995) had, in his laboratory, measured the speed of electricity to be $30.000 \mathrm{~km} / \mathrm{s}$ which is one tenth the speed of light in free space. Similarly, the study of Rakov (Y. Baba and V. A. Rakov, 2003) found the normal lightening return-stroke speed to be in the range of $2-3 * 10^{8} \mathrm{~m} / \mathrm{s}$

So, defining electrons as charge carriers which lockup charges of unknown nature violates the measured velocity of electricity. It is more plausible to accept the introduced definition of flow of electric charges as the flow of electromagnetic waves and the flow electrons as a simultaneous flow of particles and electromagnetic waves. In this case, the found velocity of electric charge is in the same order of the velocity of electromagnetic waves. Accordingly, Einstein's error during his analysis of the photoelectric effect might be asserting that the electron and charge are one entity or the electron is the electric charge.

\section{The Photon}

At the end of the 19th century, one of the most intriguing puzzles in physics involved the spectrum of radiation emitted by a hot object. Specifically, the emitter was assumed to be a blackbody as a perfect radiator. The hotter a blackbody is, the more the peak in the spectrum of emitted radiation shifts to a shorter wavelength. Nobody could explain why there was a peak in the distribution. However, Max Planck came up with the idea that a blackbody was made up of oscillating atoms, and that the energy of each oscillating atom was quantized (http://dilipkumar.in/articles/science-legends/max-planck-the-photon-of-physics.html). That last point is the key: the energy of the atoms could only take on discrete values, and these values depend on the frequency of the oscillation. Planck's predicted the emitted energy of an oscillating atom as follows:

$$
E=n h v \quad n=0,1,2,3, \ldots
$$

$v$ is the frequency, $\mathrm{n}$ is an integer, and $\mathrm{h}$ is a constant known as Planck's constant which has the value $6.6 * 10^{-34}$ $\mathrm{J}$-sec. This constant shows up in many different areas of quantum mechanics.

The spectra predicted for a radiating blackbody made up of these oscillating atoms agrees very well with experimentally-determined spectra. Planck's idea of discrete energy levels led him to consider the light waves, as a spectrum of electromagnetic waves, to flow into packets of energy called photons.. The energy of a photon is directly proportional to its frequency $v$ : 


$$
E_{p}=h v
$$

Such photons are moving at speed of light estimated as follows:

$$
c=299792458 \mathrm{~m} / \mathrm{s}
$$

According to relationship (15), a photon of light has energy of:

$$
E_{p}=2 \div 2.5 \mathrm{eV} ; 1 \mathrm{eV}=1.602176487 \times 10-19 \mathrm{~J}
$$

Assuming the behavior of such a photon as a particle; it is possible to compare momentum of a light-photon and momentum of an electron that is moving at half-light speed as follows;

$$
\frac{(m * v)_{\text {photon }}}{(m * v)_{\text {electron }}}=\frac{m_{\text {photon }}}{m_{\text {electron }}} * \frac{c}{c / 2}=\frac{2.25 * 1.602 * \frac{10^{-19}}{c^{2}}}{9.10953 * 10^{-31}} * 2=0.0000009
$$

According to the principles of momentum's conservation, this calculated ratio in equation (17) means it is required more than 1 million of the claimed light particles or photons to bounce one electron.

\section{The Photoelectric Effect}

Photoelectric effect is used to describe the process where light strikes a clean cathode plate and produce electric current in a photocell as shown in Figure 2. The explanation for the photoelectric effect goes according to Einstein like this: It takes certain energy to eject an electron from a metal surface (Albert Einstein, 1905). This energy is known as the work function (W), which depends on the metal. Electrons can gain energy by interacting with photons. If a photon has energy at least as big as the work function, the photon energy can be transferred to the electron and the electron will have enough energy to escape from the metal. A photon with energy less than the work function will never be able to eject electrons.

So, according to Einstein, when light of frequency $v$ is incident on a metal surface that has a work function $\mathrm{W}$, the maximum kinetic energy of the emitted electrons is given by (http://www.physchem.co.za/OB12-ele/photoelectric.htm):

$$
K E_{\text {max }}=h v-W
$$

Note that this is the maximum possible kinetic energy because $\mathrm{W}$ is the minimum energy necessary to liberate an electron. The threshold frequency, the minimum frequency the photons can have to produce the emission of electrons, is when the photon energy is just equal to the work function. So, the threshold frequency can be expressed as follows:

$$
v_{o}=W / h
$$

Accordingly, Einstein thought light acts as particulate in this situation (Albert Einstein, 1905). The energy is transmitted in little packets, or photons, that may behave as particles. So, he explained when a photon hits an atom, it only carries a certain amount of energy with it. Electrons must absorb more than certain amount of energy to be ejected from the atom. So, if the photon doesn't have high enough frequency, and thus high enough energy, the electron won't be ejected from the atom. It doesn't matter how many photons hit the atoms: the energy is not cumulative

The interaction between a photon and an electron is defined according to "Compton scattering" as the collision of the photon elastically with an atomic electron and transferring both momentum and energy (W. Sinnema, 1979). So, success of such collision to eject an electron out of the atom is in need to transfer both momentum and energy. According to previously found results in equation (17) and by applying the principles of conservation of momentum, it is required more than a million photons to bounce one electron if we assume the velocity of the orbiting atomic-electrons is half speed of light. So, bouncing an electron by the impact of a photon is also a concern of momentum conservation and not only of energy conservation. So, the cut-off frequency that defines only the energy of a photon should not be considered as the only criteria for the claimed ejection of electrons by such a photon. 
Reviewing the measurement plot in Figure 4, it shows how the photocurrent increases when the light intensity increases but the frequency is held constant (G. Keiser, 2000). The retarding potential that stop the flow of the reflected ionized photons is the same as the frequency of the incident light is kept constant (G. Keiser, 2000). Such results are suggesting that the reflected waves gain a specified potential that depends on its frequency and hence independent of light intensity. Such phenomena cannot be explained if we consider, according to Einstein, the light particles eject electrons. This may represent a sufficient proof of plausibility of considering the photoelectric effect is a result of converting the light-photons into energy quanta or charges of quantized potential that depends on the frequency of the incident light. Similarly, it is a proof that the charges are reflected electromagnetic waves that gain an electric potential equal to the stopping voltage in this experiment.

Similarly, the plot in Figure 5 of the measured stopping potentials obtained for several light frequencies for two different metals indicates also the dependence of the gained potential of the incident photons on the electrode potential of the reflecting metal in addition to the incident-light frequency. The slope of this plot is the same in both cases and is equal to Planck's constant "h". The $\mathrm{x}$-intercept corresponds to the lowest frequency of light or the lowest energy that can gain an electric potential during its reflection. So, the change of the value of such potential depends on the amplitude of the wave that may adopt such potential in presence of certain metal according to its electrode potential. The measured influence of the metal's electrode potential on the threshold frequency assures also the postulated nature of electric charges as reflected waves of specified electric potentials.

Results of measurements of the X-ray Photoelectric Effect are shown in Figures 5 and 6 where photon's energy in the range $1 \mathrm{keV}-0.5 \mathrm{MeV}$ hit a metal surface ( $\mathrm{S}$. Hüfner, 2003,http://www.docstoc.com/docs/80625378/X-Ray-Photoelectron-Spectroscopy-XPS). The shown quantization of photon's energy or potentials of the reflected spectrum at definite values in Figures 5 and 6 is similar to that found in the plots of Figures 3 and 4 . Such similarity may offer a proof of the introduced explanation of the photoelectric effect as photons of light gains actually specific values of quantized potential according to its frequency during its reflection in photocells (J. N. Bradford, 1973, W. Demtroder, 2006). However, other literature described such groupings or discontinuities according to the values of binding energies in different shells of atoms ( J.W. ROHLF,1994). Such description assumes photons may cross the atom's shells to eject electrons at different orbits. However, the arrangement of atoms within a crystal is determined by X rays crystallography where the atoms reflect or scatter $\mathrm{X}$-rays to assign its location inside the crystals (http://www.tulane.edu/ sanelson/eens211/x-ray.htm). Such reflections are not done by electrons as a proof that may violate such an assumption of crossing the atom's boundaries by high energy photons (P. Atkins and R. Friedman, 2008).

\section{Modified Definitions of Photoelectric effects}

In modern literatures, the photoelectric effect is defined as "one" photon is giving its energy to a single atomic electron and ionizing the atom in a single interaction (http://www.tulane.edu/ sanelson/eens211/x-ray.htm). According to Einstein, such photon should eject one electron to ionize the atom in a single interaction. However, the previous analysis of Compton Scattering indicates the significance of the number of photons. So, the previously stated definition that limits one photon to ejecting a single atomic electron and ionizing the atom needs to be modified. In other words, the photoelectric effect should be defined as "ionizing" the incident photons during its reflection instead of "ionizing" the shocked atoms due to ejecting electrons. Such definition depends on the introduced definition of the flow of electric charges as a flow of ionized photons or electromagnetic waves (S. Abdelhady, 2010, pp. 162- 166) and on measuring the photoelectric effects of electromagnetic waves of different wavelengths (G. Keiser,2000, S. Hüfner, 2003).

According to Planck analysis of energy quantization, equation (14), the electric charge as a form of energy is quantized too (D. Haaiday,et al., 2004). If we denote the elementary charge of a single electron or proton which is found experimentally as (D. Haaiday,et al., 2004):

$$
\mathrm{e}=1.60 \times 10^{-19} \text { Coulomb, (Joule/volt) }
$$

Then any positive or negative charge q has the form (D. Haaiday,et al., 2004),

$$
\mathrm{q}=\text { ne, where } \mathrm{n}= \pm 1, \pm 2, \pm 3, \ldots \quad(\mathrm{n} \in \mathrm{Z})
$$

Accordingly, the minimum photon's energy that can be converted into an electric charge is: $\mathrm{e}=1.6 \times 10^{-19}$ Joule/volt at a minimum quantized potential of 2 Volt as found by measurement in Figure 3 (G. Keiser, 2000). So, energy of the minimum charge can be found as follows: 


$$
\begin{aligned}
E=e * E= & 1.60 \times 10-19\left(\frac{\text { Joule }}{\text { volt }}\right) * 2 \text { Volt } \\
& =3.2 \times 10-19 \text { Joule }
\end{aligned}
$$

Such energy can be used to determine the photon's threshold frequency or the minimum photon's frequency that can be converted into charge according to the following equation:

$$
\begin{gathered}
E=h * v=6.626 \times 10-34 * v=3.2 \times 10-19 \quad \text { Joule } \\
\text { So, } v=0.480 \times 10^{15} \mathrm{~s}^{-1}
\end{gathered}
$$

Such photon's frequency is the frequency of blue light which is similar to the value discovered by Einstein. Hence, the defined Einstein threshold energy may not express the minimum energy that can eject an electron but the minimum energy quanta that may gain the measured electric potential of 2 Volt (G. Keiser, 2000). The found value of the minimum charge's energy in equation (20) can be used to define the electron-Volt $(\mathrm{eV})$ as half the minimum energy of an ionized photon or charge.

According to the introduced entropy approach, Einstein's explanation of photoelectric effect may be criticized in three aspects:

1). Bouncing of electron is mainly a momentum problem that requires application of principle of momentum conservation and that signifies importance of the number of the colliding or bouncing photons.

2). The reflected charges do not necessarily indicate bouncing of electrons as such charges might be ionized photons.

3). The introduced threshold frequency as discovered by Einstein may be defined as the minimum energy that can be converted into electric charge as found in measurements of Figures 3 and 4 (G. Keiser, 2000).

Finally, light should be considered as a wave only and its behavior as a particle that ejects electrons, in the photoelectric effect, may be considered as a misconception.

\section{Conclusions}

Einstein's descriptions of Electrons, Photons, and the Photo-Electric Effect are reviewed according to a recently introduced entropy approach. It is found that Einstein's hypothesis of the duality of light as the sole explanation of the photoelectric effect might be a misconception. Physically, light cannot eject electrons but the photons may be negatively ionized during their reflection due to the cell's electric field. The truth of the nature of the flow of electric charges as a flow of electromagnetic waves that have an electric potential contradicts Einstein's description of electric charges. However, truth of the introduced postulate is verified in clearing the confusions of duality of light and electrons, the drift velocity of electric charges, the conflicts in the SI system of units, the stopping voltages in photocells, the dependence of the threshold's frequency on the metal's surface, the energy or potential quantization that is found in the photoelectric effect of light at different wave lengths and in photoelectric effect of the X-rays.

\section{References}

A. H. Compton. (1923). A Quantum Theory of the Scattering of X-rays by Light Elements, The Physical Review, Vol. 21, No. 5, May. http://dx.doi.org/10.1103/PhysRev.21.483

Albert Einstein. (1905) Annal der Physik, 17,132.

Bray John. (1995). The First Telegraph and Cable Engineers in The Communications Miracle - The Telecommunications Pioneers from Morse to the Information Superhighway, Plenum Press, New York, pp 35-49

C. Yunus and A. B. Michael. (2006). Thermodynamics: An Engineering Approach, McGraw-Hill Science Engineering.

D. Haaiday, R. Resnick R. and J. Walker. (2004) Fundamentals of Physics, $7^{\text {th }}$ edition, New York: John Wiley \& Sons.

D. L. Anderson. (1964). The Discovery of the Electron, Princeton, N.J., Van Nostrand.

D.J. Griffiths. (2004). Introduction to Quantum Mechanics. Benjamin Cummings. 
E.J.S. Fonseca, C.H. Monken, and S. Pádua , Measurement of the de Broglie Wavelength of a Multiphoton Wave Packet. Phys. Rev. Lett., 82, 2868

F.A. White. (1989). The Concept of Mass, Physics Today, 42 June, pp. 31-41

G. Keiser. (2000). Optical Fiber Communication, McGraw-Hill, third edition.

H. D. Zeh, The Physical Basis of the Direction of Time, Science, Vol. 249, no. 4965, pp. 192-193.

J. N. Bradford. (1973). X-Ray Induced Electron Emission II, IEEE transactions on Nuclear Science, Volume 20, 6, 105-110. http://dx.doi.org/10.1109/TNS.1973.4327379

J.W. ROHLF. (1994). Modern Physics from a to Z, Wiley, New York.

P. Atkins and R. Friedman. (2008). Molecular and Quantum Mechanics, $4^{\text {th }}$ edition, Oxford University Press.

S. Abdelhady. (2010). Comments Concerning Measurements and Equations in Electromagnetism. $J$. Electromagnetic Analysis \& Applications, March, 2: pp. 677-678.

S. Abdelhady. (2010). A Fundamental Equation of Thermodynamics that Embraces Electrical and Magnetic Potentials, J. Electromagnetic Analysis \& Applications, March, 2: pp. 162- 166.

S. Abdelhady. (2010). An Approach to a Universal System of Units. J. Electromagnetic Analysis \& Applications, March, 2: pp.549-556.

S. Abdelhady. (2011). An Entropy-Approach to the Duality Property J. Electromagnetic Analysis \& Applications, March, 3: pp.220-227.

S. Hüfner. (2003). Photoelectron Spectroscopy Principles and Applications, Springer, 3rd edition.

W. Demtroder. (2006). Atoms, Molecules and Photons, Springer.

W. Sinnema. (1979). Electronic Transmission Technology, The Prentice-Hall.

W.M. Yao. (2006). Review of Particle Physics. Journal of Physics, G 33 (1): 77-115.

Y. Baba and V. A. Rakov. On the transmission line model for lightning return stroke GEOPHYSICAL RESEARCH LETTERS, Vol. 30, No. 24, 2294. http://dx.doi.org/10.1029/2003GL018407

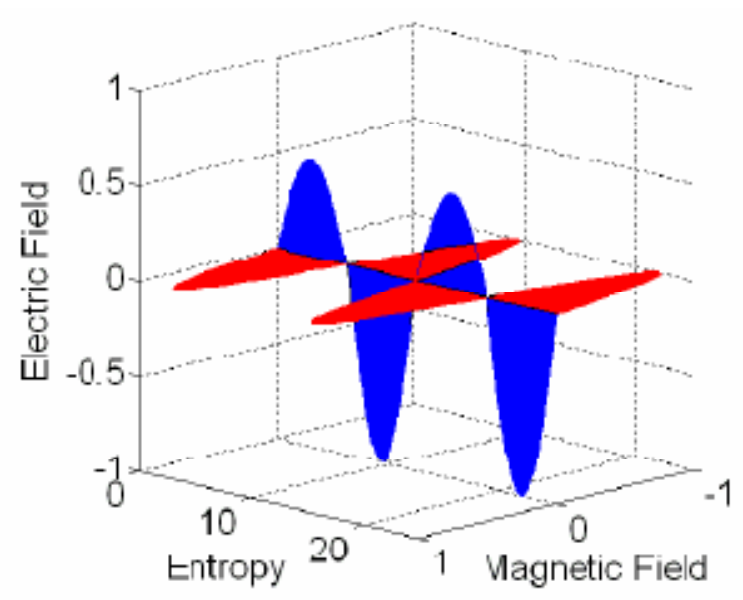

Figure 1. Flow of electric charges as electromagnetic waves of -ve potential (S. Abdelhady, 2010, pp. 162-166) 


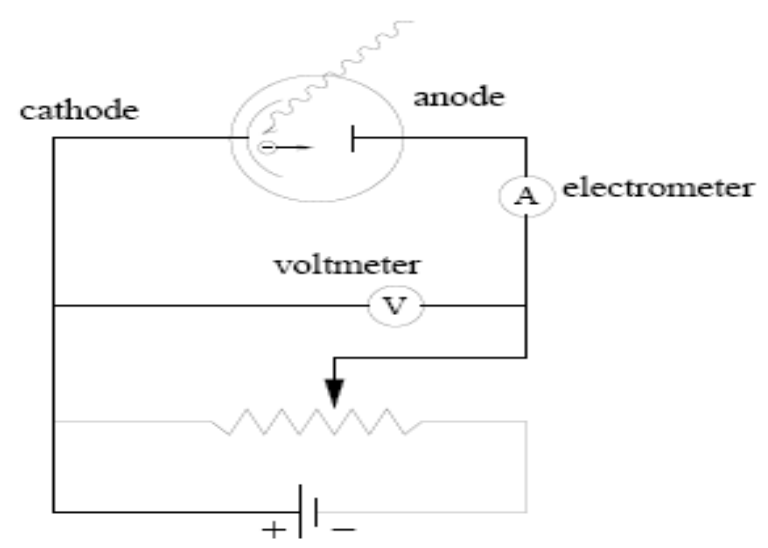

Figure 2. Photoelectric Effect Schematic

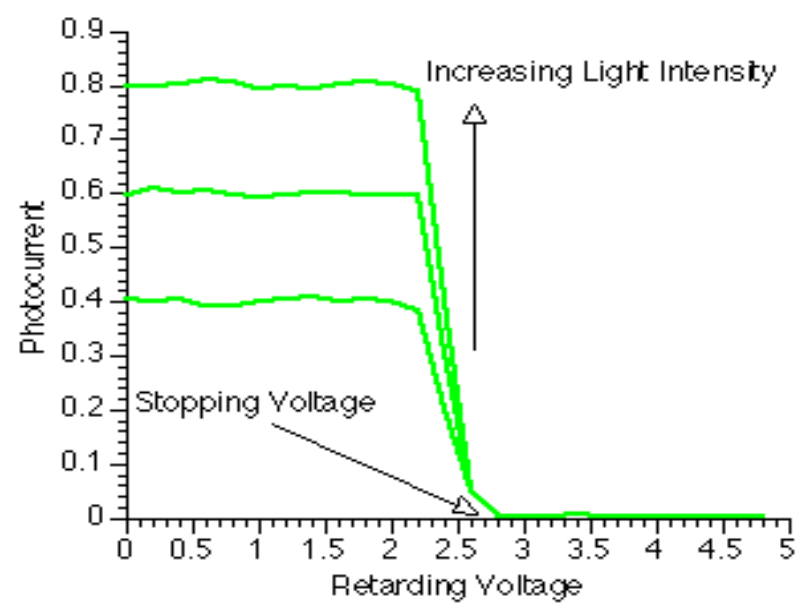

Figure 3. Measurement results of the dependence of the photocurrent on light intensity (G. Keiser, 2000)

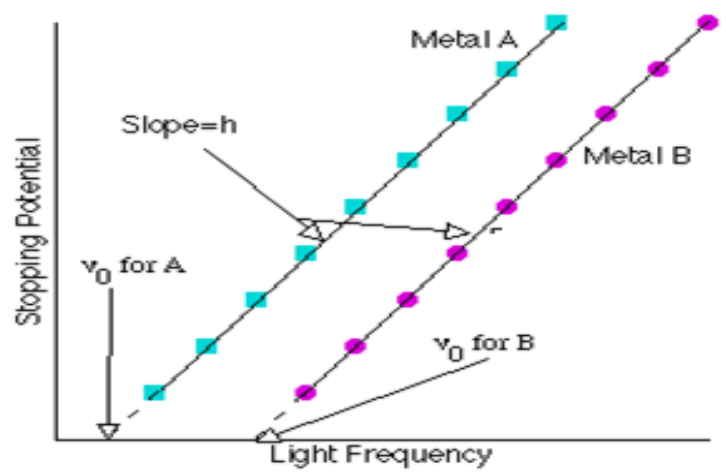

Figure 4. Plot of the measured stopping potentials obtained for several light frequencies for two different metals (G. Keiser, 2000) 


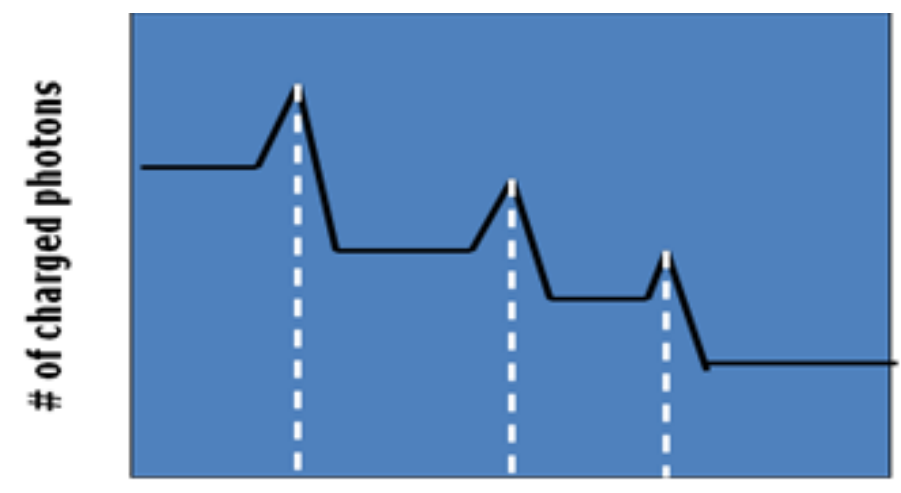

Quantized potential

Figure 5. A diagram to identify that the charges are grouped at quantized potentials in Volts or energies in $\mathrm{eV}$

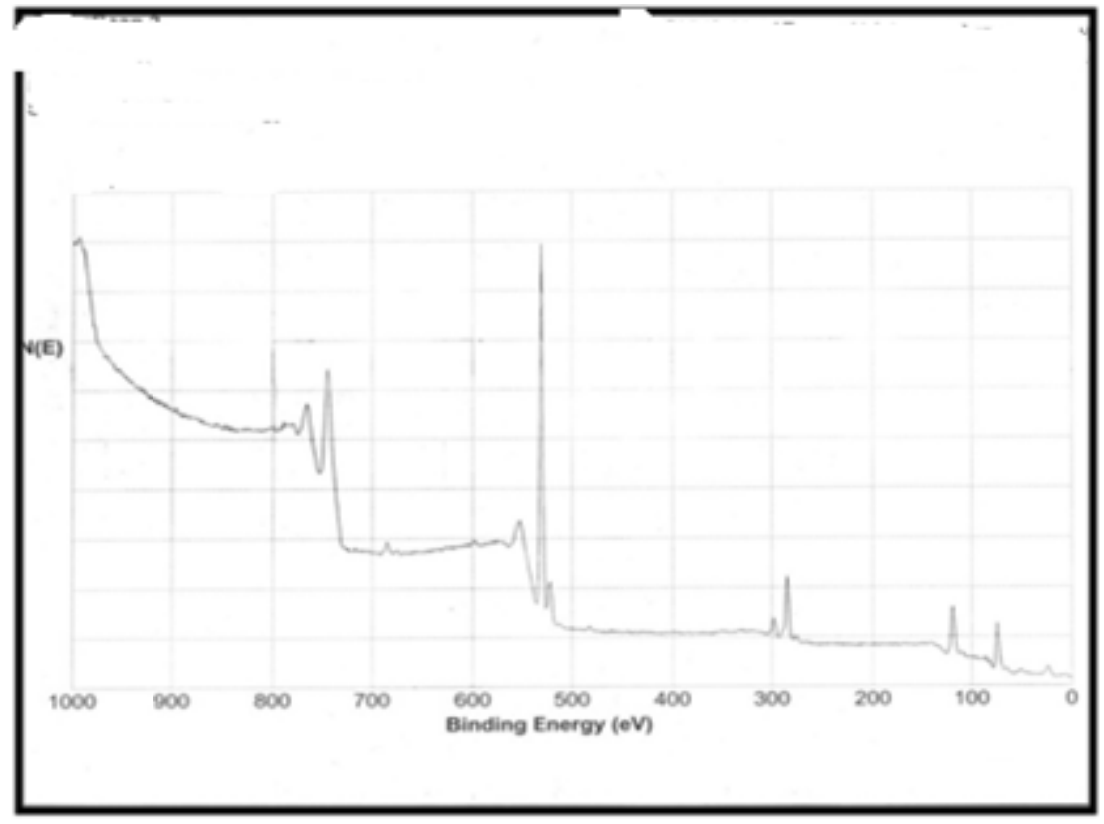

Figure 6. Spectrum resulting from incidence of $\mathrm{x}$-ray photons on a metal surface inside a photocell (J. N. Bradford, 1973) 